\title{
Cooperación internacional para el desarrollo de energías renovables: energía eólica y solar en el
}

mundo

International cooperation for the development of renewable energies: wind and solar energy in the world

Alejandro, Castillo-Maldonado ${ }^{1}$

Universidad Autónoma de Nuevo León

\section{RESUMEN}

El presente artículo es producto de una revisión bibliográfica, cuyo objetivo consistió en mostrar evidencia del estado de la cooperación internacional para el desarrollo de las energías renovables, particularmente la energía eólica y solar. Se aplicó el método de análisis, con un enfoque cualitativo, diseño no experimental, bajo un nivel documental-bibliográfico transversal. Tras la revisión documental se encontró que: Debido a la disminución en las reservas internacionales y caída de los precios del petróleo, las energías renovables (ER), no sólo pueden ser una opción de un desarrollo sustentable, sino también pueden ser fuente de nuevos empleos e innovación. En el presente capitulo se abordará de manera general. Se concluye que se requiere determinar las características de las energías renovables en el mundo, así como las acciones que han tomado diversos países para promoverlas de manera local y a través de la cooperación internacional.

Palabras clave: Cooperación internacional, desarrollo, sustentabilidad global, energía renovable, energía eólica, energía solar.

\section{ABSTRACT}

This article is the product of a literature review, whose aim consisted in showing evidence of the international cooperation status for the development of the renewable energies, especially solar and wind energies. The method of analysis was applied with a qualitative approach, non-experimental design, under a documentary-literature cross sectional level. After the documentary review, it was found that: due to the decrease on the international reserves and the drop on oil prices, renewable energies (RE) can not only be a sustainable option, but can also be a source of innovation and new jobs. In this chapter it will be reviewed in a general way. It is concluded that it is necessary to determine the renewable energies characteristics in the world, likewise the actions that several countries have taken to promote them in a local way and through the international cooperation.

Key words: International cooperation, development, global sustainability, renewable energy, wind energy, solar energy.

\section{Cómo referenciar este artículo:}

Castillo-Maldonado, A. (2017). Cooperación internacional para el desarrollo de energías renovables: energía eólica y solar en el mundo. Política, Globalidad y Ciudadanía, 62-72. http://revpoliticas.uanl. $\mathrm{mx} /$ index.php/RPGyC/article/view/80

Recibido: 14 de Febrero 2017 - Aceptado: 18 de Abril 2017

(cc) BY-NG-ND

1 Maestría en Relaciones Internacionales por Universidad Autónoma de Nuevo León, Email: alejandro.fpoliticas@gmail.com

Revista Política, Globalidad y Ciudadanía, Vol. 3 No. 6, Julio - Diciembre 2017, Universidad Autónoma de Nuevo León, Monterrey, México Monterrey, México, ISSN 2395-8448. pp 62-72. http://revpoliticas.uanl.mx/index.php/RPGyC/article/view/80 


\section{1.- INTRODUCCIÓN}

La sustentabilidad global y el desarrollo de nuevas alternativas energéticas están entrelazados a la cooperación internacional para el desarrollo, es por ello que resulta importante determinar el estado de las energías renovables en el mundo, particularmente la energía eólica y solar, así como las posibilidades de que se traduzcan en desarrollo. Se propone determinar las características de las energías renovables en el mundo, debido a que "la finitud de las fuentes fósiles y su impacto al medio ambiente. La estructura energética mundial es no sustentable y se requiere de un cambio de paradigma energético basado en la eficiencia energética y el uso de fuentes alternas como las energías renovables (ER). La demanda energética mundial está en continuo aumento a un ritmo de crecimiento anual del 2.47\%” (Estrada, 2013). Es así que las acciones que han tomado diversos países para promoverlas de manera local y a través de la cooperación internacional son de importancia para analizar la situación actual y el futuro de las ER.

Durante las últimas décadas ha cobrado importancia significativa el interés por el medio ambiente, como resultado de una preocupación genuina sobre el estado que guarda el planeta, "los costos de adaptación al cambio climático serán colosales: sugiere que para 2030, el mundo tendrá que gastar más de 200,000 millones de euros anuales en medidas como construir barreras de defensa contra inundaciones, transportación de agua para agricultura y reconstrucción de infraestructura afectada por fenómenos climáticos" (Parry et al. 2009)2.

Temas como medio ambiente, calentamiento global, efecto invernadero, contaminación son escuchados por los diversos medios informativos y estudiados desde cualquier ámbito relacionado con la ciencia y la academia; incluso se han creado carreras universitarias como las de ingeniería ambiental, administración de la energía y desarrollo sustentable entre otras, las cuales buscan formar profesionistas e investigadores en la materia.

El clima depende de un gran número de factores que interactúan de manera compleja. (...) Cuando un parámetro meteorológico como la precipitación o la temperatura sale de su valor medio de muchos años, se habla de una anomalía climática ocasionada por forzamientos internos, como inestabilidades en la atmósfera y/o el océano; o por forzamientos externos, como puede ser algún cambio en la intensidad de la radiación solar recibida o incluso cambios en las características del planeta (concentración de gases de efecto invernadero, cambios en el uso de suelo, etc.) resultado de la actividad humana. (Magaña, 2004)

La importancia creciente de los problemas de seguridad energética y la cooperación internacional en el sector de la energía ha fomentado los esfuerzos por concretar una energía sin fronteras, es decir, cooperación internacional en energías renovables y tecnología verde. Uno de los problemas más importantes a los que se enfrentan tanto la comunidad internacional como el derecho internacional es la reconciliación energética y ambiental. Los precios inestables, el calentamiento global, la dependencia de las importaciones de energía y los crecientes costos de producción hacen que sea necesario desarrollar nuevas fuentes de energía. Las economías más importantes quieren salvaguardar su seguridad energética en tecnologías de bajas emisiones. Entonces, una posible solución al problema del fortalecimiento de la seguridad energética internacional y el desarrollo sostenible es el uso de tecnologías verdes y fuentes de energía renovables. Se requiere determinar qué puede hacer la comunidad internacional para materializar la idea de la solidaridad en el ámbito de la energía, las acciones que deberían tomarse para apoyar el desarrollo de fuentes de energía renovables, los temas de las nuevas tecnologías energéticas; las fuentes de energía renovables, incluida la energía eólica y los nuevos combustibles para el transporte. La seguridad energética y la cooperación internacional, nuevas políticas ambientales y tecnologías verdes; y un nuevo tema, la seguridad

$2 \quad$ Traducción libre del autor

Revista Política, Globalidad y Ciudadanía, Vol. 3 No. 6, Julio - Diciembre 2017, Universidad Autónoma de Nuevo León, Monterrey, México Monterrey, México, ISSN 2395-8448. pp 62-72. http://revpoliticas.uanl.mx/index.php/RPGyC/article/view/80 
y gobernanza energética.

\section{2.- FUNDAMENTO TEÓRICO}

Energías renovables en el mundo: energías eólica y solar

Sin bien la preocupación ha llevado a los gobiernos de los países y a las organizaciones de la sociedad civil y organismos no gubernamentales a desarrollar mecanismos de cooperación y desarrollo internacional en materia ambiental, que buscan contrarrestar el fenómeno del calentamiento global, a esto también se le puede sumar la reciente situación de la industria petrolera, cuyos precios han mostrado una caída significativa en el último trienio, provocando una posible crisis energética de hidrocarburos global. "Este contexto de declinación de las reservas del "petróleo fácil" es ya evidente. Muchos de los campos de petróleo y gas del mundo están llegando a su madurez" (Estrada, 2013).

En general todos los países del mundo, ya sean desarrollados como Alemania, Estados Unidos y Rusia; o los también llamados Small States como Dinamarca, Estonia e Islandia, y los países emergentes como Brasil, China, Colombia y México necesitan para su desarrollo e inclusión en la arena internacional desarrollar una infraestructura que incluya cada vez más a las energías modernas, debido a los altos consumos energéticos de sus poblaciones en la actualidad e inclusive como estrategia de seguridad energética nacional y como parte de su política exterior y como medio de defensa ante el actual ambiente de seguridad internacional. Es por esta razón que "la instalación y uso de Fuentes No Convencionales de Energía (FNCE), tales como la energía eólica y la fotovoltaica, al igual que todo proyecto que se ejecute en una región, tienen asociados unos impactos ambientales, sociales y económicos que se deben evaluar con el $\mathrm{n}$ de tomar decisiones tales como el lugar de ubicación del proyecto, el tamaño de la instalación, etc. Con el fin de considerar todos los posibles impactos de un proyecto de este tipo se deben evaluar los impactos en las 3 fases principales de su ejecución: construcción, operación y desmantelamiento" (Pasqualino et al. 2015).

De acuerdo a IEA, (2014) y REN21 (2014) citado en Beltrán Téllez et al. (2017):

El papel de la energía solar puede pasar de ser un margen pequeño de contribución, como se considera actualmente, a llegar a ser la más importante fuente energética en el 2050, debido a que este tipo de energía tiene el potencial más amplio de todo el portafolio de energías disponibles, con un $40 \%$.

La energía del viento o eólica, está basada indirectamente en la energía del sol. Una pequeña proporción de la radiación solar recibida por la Tierra se convierte en energía cinética. La causa principal es el desequilibrio entre la salida de energía neta de altas latitudes y la entrada de energía neta a bajas latitudes. La rotación de la Tierra, características geográficas y gradientes de temperatura, afectan la localización y naturaleza del viento requiere que la energía cinética se transforme en energía mecánica (movimiento de las aspas) y ésta en energía eléctrica, a través de generadores, para que llegue a ser útil.

Para comprenderlo de una mejor manera, "el viento, que es el aire en movimiento, es causado principalmente por el efecto térmico en capas bajas de la atmósfera. En la capa próxima a la superficie terrestre, hasta $15 \mathrm{~km}$, llamada troposfera, el aire es calentado indirectamente por la radiación solar. Esta energía es absorbida por la tierra e irradiada a la troposfera, que absorbe una parte. Las diferencias térmicas provocan, a su vez, diferencias de presión que originan la circulación de masas de aire facilitando el intercambio de calor entre el ecuador y los polos" Brizuela (2004).

Sin duda la energía solar en sus distintas formas (directa e indirecta) es la energía renovable más

Revista Política, Globalidad y Ciudadanía, Vol. 3 No. 6, Julio - Diciembre 2017, Universidad Autónoma de Nuevo León, Monterrey, México Monterrey, México, ISSN 2395-8448. pp 62-72. http://revpoliticas.uanl.mx/index.php/RPGyC/article/view/80 
abundante, ya que puede aprovecharse desde la radiación solar directa o difusa hasta la energía eólica, hidráulica y biomasa.

Existen diversas situaciones que han propiciado la búsqueda de nuevas fuentes de energía, de acuerdo a Estrada (2013):

Entre los factores que han permitido el desarrollo de los mercados de las Fuentes Renovables de Energía (FRE), se pueden mencionar a los siguientes:

- El alza de los precios de los hidrocarburos que llegó a 139 USD por barril en junio del 2008 y que podrían llegar a los 150 dólares por barril o más en los años venideros.

- El mercado mundial de emisiones de CO2 que está en 19 USD la tonelada y en el futuro podría llegar hasta los 40 o 60 dólares la tonelada.

- Las políticas voluntarias de varios Estados (Unión Europea, Estados Unidos, China, India, Brasil) más iniciativas locales que crean incentivos especiales para usar tecnologías FRE.

- El progreso acelerado que han tenido las tecnologías renovables.

Figura 1. Capacidad total de generación eléctrica mundial, 2007-2016 (MW)

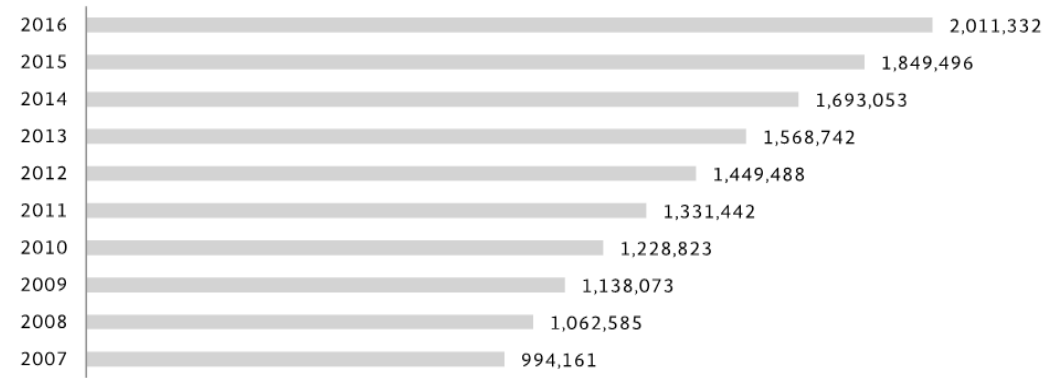

Fuente: Secretaría de Energía (SENER), México.

Como se ha mencionado efectivamente muchos gobiernos de países se han inclinado por establecer políticas públicas con el fin de proteger al medio ambiente, y esto ha generado un incremento en la participación de la capacidad instalada de energías renovables a nivel mundial. 
Figura 2. Participación de las energías renovables en la capacidad instalada mundial, 2007 y 2016

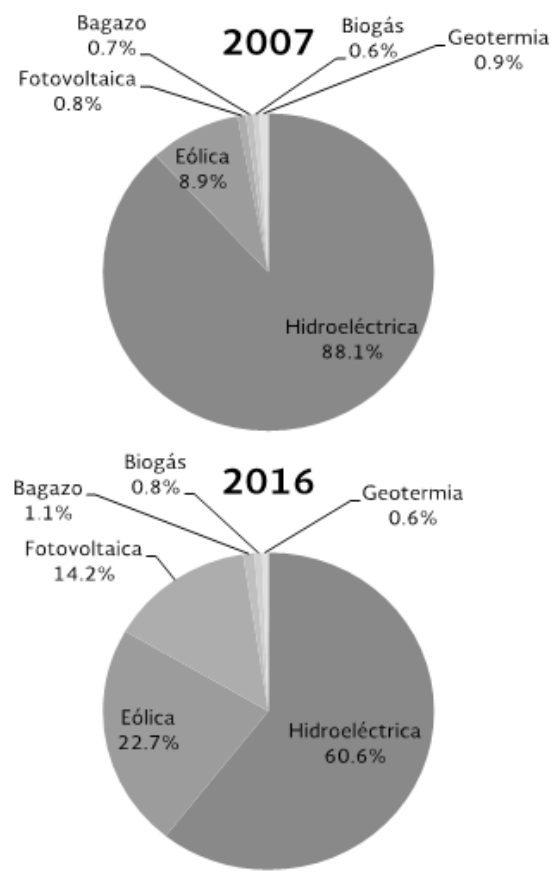

Fuente: Secretaría de Energía (SENER), México.

\section{Cooperación internacional para el desarrollo de las energías renovables}

La cooperación internacional en materia de combate al cambio climático en la actualidad y como resultado de la preocupación por el medio ambiente ha propiciado la creación de mecanismos de cooperación internacional que buscan contrarrestar los efectos dañinos al medio ambiente, entre ellos el Consejo Mundial de Energía y su programa para los futuros líderes, Panel Intergubernamental sobre el Cambio Climático, PIIC, la Convención Marco de las Naciones Unidas sobre el Cambio Climático, el Protocolo de Kioto y la Conferencia de las Partes de París en 2015, "cuyo objetivo es lograr un acuerdo universal, jurídicamente vinculante, para reducir las emisiones de gases de efecto invernadero a fin de limitar el aumento de la temperatura global del planeta a $2{ }^{\circ} \mathrm{C}$ respecto a los niveles preindustriales. La Conferencia de París constituye la culminación de un largo proceso de negociación, arduo y difícil, para renovar los compromisos asumidos por las Partes en 1997. Como en anteriores Conferencias, su conclusión exitosa requerirá grandes dosis de trabajo, paciencia y habilidad negociadora, puesto que el proceso de negociación ha sufrido un desgaste importante a lo largo de estos años, especialmente desde 2009. La situación ya no es la misma que llevó a finales de los noventa a subscribir de modo entusiasta el Protocolo de Kioto. De hecho, desde su entrada en vigor, todo el proceso se encuentra lastrado por la falta de apoyo y de confianza entre las Partes, 4 especialmente de los actores principales en la escena internacional." (Campins, 2015)

Es importante mencionar que para poder continuar con las metas establecidas en diversos foros internacionales y al ritmo que se ha incrementado en las últimas décadas, es necesario seguir invirtiendo en $\mathrm{I}+\mathrm{D}+\mathrm{E}+\mathrm{i}+\mathrm{e}$, es decir, investigación y desarrollo, educación, e innovación y emprendimiento, ya que es a través de la convergencia de estas, incluyendo la creación de empresas y empleos en el sector, generando el desarrollo tecnológico y económico que propicia el crecimiento.

Beltrán Téllez et al. (2017) menciona que "en cuanto al desarrollo tecnológico, los 10 principales fabricantes de turbinas, que capturaron el $73.2 \%$ de ventas del mercado mundial en 2015, frente al $77 \%$ 
en 2012, se distribuyeron de la siguiente manera: China (5), Europa (4), y EE. UU. (1). En el 2015, China dominó el mercado con el $28 \%$, seguida por Dinamarca con $11.8 \%$ y EE. UU. con un $9.5 \%$ (REN21, 2015; REN21, 2016). En el 2015, la marca Goldwind obtuvo el primer lugar en ventas, con un $12.5 \%$, Vestas el segundo con el 11.8 \%, GE Wind el tercero y Siemens obtuvo el cuarto lugar (REN21, 2014; REN21, 2015; REN21, 2016)."

La cooperación internacional, como ya se ha mencionado incluye no sólo la cooperación entre Estados, sino también entre organizaciones no gubernamentales ( $\left.\mathrm{ONG}^{\prime} \mathrm{s}\right)$, empresas, universidades, individuos y organizaciones internacionales, incluyendo las principales actividades de la población, tal como menciona el Consejo Mundial de Energía (2014):

En colaboración con otras organizaciones internacionales, como la Organización Mundial de Comercio (OMC), el CME aspira a influir en los debates a nivel regional e internacional para mejorar el acceso a los mercados y superar las barreras comerciales transnacionales a las que se enfrentan los bienes y servicios energéticos. Las recomendaciones de nuestras Reglas para el comercio ya han sido aprovechadas por los miembros del Foro de Cooperación Económica Asia-Pacífico, quienes han acordado poner un límite del 5\% a las tasas arancelarias para 54 tipos de bienes "ecológicos".

En este caso el comercio internacional afecta el cambio climático debido al consumo de energía derivada de la transportación de insumos y mercancías, así como la emisión de $\mathrm{CO} 2$ por los medios de transporte utilizados, y este proceso incluye la logística inversa, para reciclaje o por defectos en su producción, provocando un proceso extra en el que también se consume energía y se genera contaminación por transporte y almacenaje.

Acciones que se han tomado en el mundo para promoverlas de manera local: los casos de Dinamarca, España y Estonia

Aunque existen países en los que el fenómeno del cambio climático se ha tomado muy seriamente y han mostrado grandes avances en la búsqueda, desarrollo y utilización de fuentes de energía renovables como la eólica y la solar, también hay países que se han quedado rezagados o bien que aún se desconoce el potencial que tienen en esta materia, como es el caso de Rusia, que, "con poca experiencia rusa en este sector, escaso conocimiento tecnológico sobre las energías renovables y estructuras inadecuadas del mercado interno, el desarrollo de un sector nacional de bioenergía sólida no es nada fácil. Por lo tanto, los desarrollos de pellets de madera del noroeste de Rusia no pueden remontarse a nuevas políticas federales, solo en parte a la demanda local y la estimulación, y significativamente a los conductores extranjeros. La gran demanda de la UE de pellets de madera y la intensificación de la colaboración con empresas extranjeras y organizaciones líderes en el campo de la investigación, la tecnología y el comercio de bioenergía sólida han desencadenado estos desarrollos. Pero es un largo camino antes de que Rusia sea liberada de su adicción a los combustibles fósiles, como reiteradamente prometieron los funcionarios gubernamentales" (Pristupa y Mol, 2015).

\section{El caso de Dinamarca.}

Uno de los países líderes en la generación de energía eólica en el mundo es Dinamarca, de acuerdo a la Asociación Danesa de la Industria Eólica (2014):

Una mirada rápida a los números lo dice todo: en Dinamarca, las turbinas eólicas son una parte vital del sistema energético, ya que proporcionan alrededor del 39\% de la electricidad danesa en 2014. En enero de 2014, las turbinas eólicas entregaron un promedio del $63 \%$ del consumo total. Hoy, Dinamarca usa la mayor parte de la energía eólica por ciudadano en el mundo. El porcenta- 
je récord destaca la posición de Dinamarca como líder en la búsqueda del sistema energético más renovable y competitivo en costos del futuro.

Dinamarca es un ejemplo en generación de energía eólica, además de ser uno de los pioneros en el mundo, sigue buscando ser referencia en el sector, y se espera que para el 2022 la generación combinada de energías renovables alcance el $70 \%$ de la energía danesa.

\section{El caso de España}

España es sin duda alguna uno de los países en el mundo en donde el desarrollo de energías renovables, principalmente la eólica y solar han tenido un éxito incomparable, ya que ha contribuido también a la creación de empleos en un momento de crisis económica interna, y al ser también un país exportador de generadores de energía eólica y paneles solares ha provocado el ingreso de divisas extranjeras.

Jiménez (2011) menciona que:

En relación a la meta de energías renovables, a mediados del año 2005 el gobierno español publicó el "Plan de Fomento de las Energías Renovables 2005-2010", el cual buscaba revisar las metas fijadas en el plan anterior (período 2000-2010), además de definir nuevas acciones para alcanzar dichos objetivos. El objetivo principal era que a finales del 2010 las energías renovables cubrieran el $12 \%$ del consumo primario energético, pero también se buscaba que estas energías produjeran el $30,3 \%$ del consumo bruto de electricidad y proveyeran el 5,8\% de la energía utilizada en el transporte.

Sin embargo, a pesar de este éxito, es preciso que se mantenga e incremente la capacidad española de generación de energía eólica y solar para no quedar rezagada de los nuevos países que están invirtiendo exitosamente en este tipo de energías.

\section{El caso de Estonia.}

La capacidad de generación de energía eólica en el Mar Báltico ha sido aprovechada por varios países nórdicos como Noruega, Suecia, Finlandia y por supuesto Dinamarca cuyo caso ya se ha expuesto en este trabajo de investigación. Sumado a ellos, los Países Bálticos, Lituania, Letonia y Estonia han iniciado desde hace tiempo también sus respectivos proyectos de generación de energía eólica, siendo también pioneros en la cooperación internacional en esta materia. De acuerdo al Ministerio de Asuntos Económicos y Comunicación de la República de Estonia (2015), "se han establecido mecanismos de cooperación acorde con la Directiva Europea (2009/28/EC, Art. 6-8), en el cual se establece que cualquier Estado Miembro puede conjuntamente con otro colaborar para alcanzar sus propias metas de generación de energía renovable. Es decir que pueden solicitar a otro Estado Miembro que produzca energía renovable para ellos financiándole las instalaciones ofreciendo como ventaja para el Estado productor mejorar en sus estadísticas ambientales." Menciona también lo siguiente:

- El objetivo de energía renovable de la UE 2020 para Estonia es del 25\%.

- En 2006, la participación de la energía renovable en el consumo final bruto de energía de Estonia fue del $16 \%$.

- Hoy se espera que Estonia tenga un excedente de energía renovable hasta el año 2020 ya que la participación de ER alcanzó el 25\% ya en 2011.

- Experiencia y confianza en el comercio internacional de emisiones.

- Entre 2010-2011, más del 50\% de las ventas de cuotas gubernamentales de CO2 (AAU) del excedente del mundo se han hecho por Estonia. Durante 2010-2013, las ventas de AAU-s por parte de Estonia ascendieron a 392 millones de EUR.

- Impulsor pionero en la Implementación Conjunta (JI). Estonia ha implementado 12 proyectos

Revista Política, Globalidad y Ciudadanía, Vol. 3 No. 6, Julio - Diciembre 2017, Universidad Autónoma de Nuevo León, Monterrey, México Monterrey, México, ISSN 2395-8448. pp 62-72. http://revpoliticas.uanl.mx/index.php/RPGyC/article/view/80 
exitosos en cooperación con Finlandia, Austria, Suecia y la Nordic Environment Finance Corporation.

- Se adoptará pronto una base jurídica para permitir las negociaciones de los mecanismos cooperativos de ER con otros Estados miembros de la Unión Europea.

- Ecofys: un consorcio creado por la Comisión Europea ha explorado la implementación práctica de mecanismos de cooperación ER. De sus cinco estudios de caso, uno fue sobre la Transferencia Estadística entre Estonia y Luxemburgo.

Con ello se han establecido ya diversas granjas eólicas en el territorio de Estonia, que goza de un litoral al Mar Báltico y una multitud de islas, muchas de ellas deshabitadas que sirven de sitio perfecto para continuar con el ambicioso plan estonio en materia energética. Cabe mencionar que al ser Estonia un país que en su historia reciente ha alcanzado un alto nivel tecnológico, gracias a sus políticas de promoción de las nuevas tecnologías y fomento a la cultura del emprendimiento, en el cual incluso el acceso a internet de alta velocidad es gratuito y establecido como un derecho ciudadano básico en su propia Constitución.

Entonces al ser un país interconectado resulta de importancia vital, y parte de su seguridad nacional contar con un sistema de energía moderno, eficiente, seguro y autosuficiente, como es el caso de la energía eólica.

Figura 3. Energía eólica en Estonia: Granjas eólicas funcionando en el país.

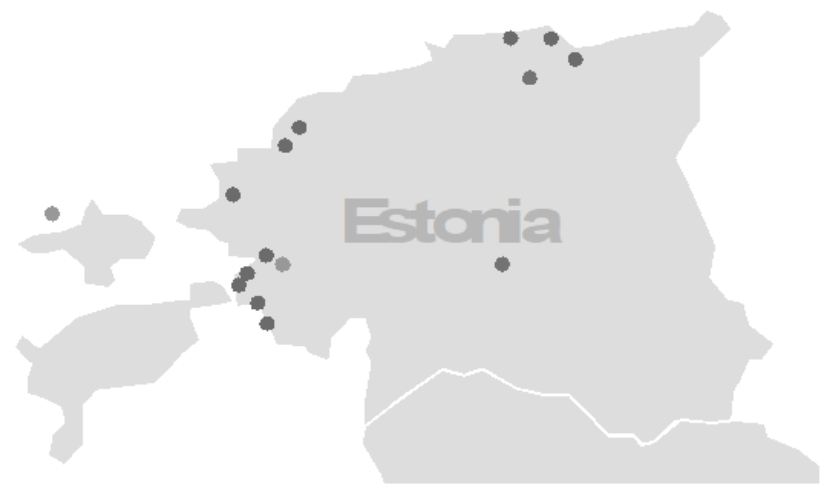

Fuente: Ministerio de Asuntos Económicos y Comunicación, República de Estonia

Contribución de las energías renovables y la cooperación internacional para el desarrollo: Proyecto de la Agencia Islandesa de Cooperación Internacional para el Desarrollo (ICEIDA, por sus siglas en inglés), de saneamiento de agua en Malawi.

La cooperación internacional abarca no sólo el proceso de promoción, investigación y desarrollo de energías renovables, sino también el desarrollo de comunidades vulnerables mediante la utilización de fuentes de energía renovable, como es el caso del proyecto islandés de agua y saneamiento en el distrito de Mangochi, Malawi:

Un proyecto que puede ser un pionero para proyectos similares en otras áreas en el futuro, ya que afecta positivamente en la vida cotidiana local. En cooperación con el gobierno de Malawi, ICEIDA gestiona un proyecto de agua y saneamiento en el lago Malawi en el distrito de Mangochi, Nankumba, con el principal objetivo de mejorar el acceso al agua potable y mejores instalaciones sanitarias para prevenir el estallido de enfermedades relacionadas con el agua como el cólera. El sistema de agua en Mangochi está alimentado con energía solar, y existen grandes 
expectativas de que dichos sistemas en el África subsahariana rural se alimentarán de esa manera (ICEDA, 2009).

El ejemplo de Islandia en materia de cooperación internacional para el desarrollo utilizando fuentes de energía renovable, en este caso de energía solar, no solamente fomenta la creación de energía, sino que a su vez ayuda a mejorar las condiciones de vida de la población de un país en desarrollo utilizando la energía solar como instrumento para el saneamiento de agua, lo cual sirve de ejemplo para otros países que buscan cooperar con otros en el desarrollo y utilización de energías renovables.

\section{3.- MÉTODO}

\section{Diseño}

El enfoque investigativo de la presente investigación es cualitativo, de acuerdo con Hernández, Batista y Fernández (2014) "Utiliza la recolección y análisis de los datos para afinar las preguntas de investigación o revelar nuevas interrogantes en el proceso de interpretación" (p.7).

Alcanzando un diseño no experimental "Que se realiza sin la manipulación deliberada de variables y en los que sólo se observan los fenómenos en su ambiente natural para después analizarlos" (Hernández, Batista y Fernández, 2014, p. 149).

El alcance establecido es el exploratorio "emplean cuando el objetivo consiste en examinar un tema poco estudiado o novedoso" (Hernández, Batista y Fernández, 2014, p. 91).

\section{Instrumentos}

Para la construcción del marco teórico-conceptual del estado de la cooperación internacional para el desarrollo de las energías renovables, particularmente la energía eólica y solar, se consultaron un total de diecisiete referencias bibliográficas utilizándose como instrumento las ideas, argumentos y proyectos que fueron interpretados desde una perspectiva analítica y crítica.

\section{Procedimiento}

Con relación a la comprensión del problema de la investigación se recopilan fuentes secundarias de documentos académicos. En el marco de referencia se definen los conceptos básicos relativos al estado de la cooperación internacional para el desarrollo de las energías renovables, particularmente la energía eólica y solar. Una vez recopilada y analizada la información se construye el documento objeto de este trabajo. Por último, se realizan las recomendaciones y conclusiones conforme a los objetivos trazados (Bascón et al, 2016, p. 39).

\section{CONCLUSIONES}

La tendencia mundial actual de energía verde y la inversión sustancial en tecnología de energía limpia está proporcionando un rápido aumento en las oportunidades de cooperación internacional y de negocios para los especialistas en la materia que incluye no solamente a los gobiernos de los distintos países del mundo, sino también a organizaciones internacionales, investigadores, tecnólogos, científicos, operadores, empresas productoras de equipos de generación de energía eólica y paneles solares, empresas especialistas en carga de proyectos que tienen las capacidades requeridas en la gestión de la cadena de suministro de equipos de gran tamaño.

De acuerdo a Jiménez (2016), "el cambio climático es un tema de interés global y local. Para ser analizado de manera holística es necesario su énfasis en la parte de la economía y su gobernanza que implica consideraciones científicas y prácticas". Es por esta razón que resulta primordial incrementar no

Revista Política, Globalidad y Ciudadanía, Vol. 3 No. 6, Julio - Diciembre 2017, Universidad Autónoma de Nuevo León, Monterrey, México Monterrey, México, ISSN 2395-8448. pp 62-72. http://revpoliticas.uanl.mx/index.php/RPGyC/article/view/80 
sólo la cooperación internacional en sector de energías renovables, sino también la investigación científica del mismo. Durante este trabajo de investigación se han encontrado dificultades por la poca información confiable y actual sobre un tema que resulta de importancia relevante para el desarrollo sustentable del mundo; se ha encontrado también que, si bien existen en el mundo diversos mecanismos de cooperación internacional puestos en marcha desde la década de 1970 incluso, tales como redes temáticas, acciones que incluyen $\mathrm{I}+\mathrm{D}+\mathrm{E}+\mathrm{i}+\mathrm{e}$ (Investigación, Desarrollo, Educación, Innovación y Emprendimiento) que coadyuvan a la investigación y transferencia tecnológica y mejora de competitividad y colaboración entre las empresas y países líderes en el sector de energías renovables, eólica y solar, primordialmente. Aún se requiere de una mayor y más estrecha cooperación entre los distintos actores internacionales y nacionales previamente mencionados para poder alcanzar las metas establecidas en los diversos foros internacionales, así como plantear retos todavía más ambiciosos como los que algunos países ya se han estado implementando, como son los casos de China, Dinamarca, Estonia, Estados Unidos, Islandia y México.

En este sentido se puede ver que en muchos países siguen mencionando las ER como una tarea pendiente a futuro, y siguen basando su economía y desarrollo en fuentes energéticas no renovables, las cuales se basan en modelos energéticos que pronto podrían ser obsoletos por el alto costo económico y por su impacto negativo al medio ambiente.

\section{REFERENCIAS}

Bascón, M.: Cazallo, A.; Lechuga, J. \& Meñaca, I. (2016). Estudio de la necesidad de implantar un servicio público de transporte entre las ciudades de Ceuta-Tetuán y Melilla-Nador. En Desarrollo Gerencial Revista de la Facultad de Ciencias Económicas, administrativas y contables de la Universidad Simón Bolívar 8(2), 37 - 57

Beltrán-Téllez, Aurelio, Morera-Hernández, Mario, López-Monteagudo, Francisco Eneldo, VillelaVarela, Rafael (2017). Prospectiva de las energías eólica y solar fotovoltaica en la producción de energía eléctrica. Ciencia UAT [[en línea] 2017, 11 (enero-junio) Recuperado de: <http://www. redalyc.org/articulo.oa?id=441949672008 $>$ ISSN 2007-7521

Brizuela, Armando B., Aguirre, César (2004). Energía no convencional -solar y eólica- para escuelas rurales en la Provincia de Entre Ríos. Ciencia, Docencia y Tecnología [en linea] 2004, XV (mayo). Recuperado de: http://www.redalyc.org/articulo.oa?id=14502807> ISSN 0327-5566

Danish Wind Industry Association (2014). Denmark-Wind Energy Hub, Profile of the Danish Wind Industry. Frederiksberg, Denmark. Recuperado de: http://ipaper.ipapercms.dk/Windpower/ Englishpublications/Denmark_Wind_Energy_Hub/ [Fecha de consulta: 4 de enero de 2018]

Estrada Gasca, C.A. (2013). Transición energética, energías renovables y energía solar de potencia. Revista Mexicana de Física, 59 (2), 75-84.

Hernández, R., Fernández, C., y Baptista, P. (2014). Metodología de la investigación. México: Mc Graw Hill.

Icelandic International Development Agency (2009). Malawi: The Icelandic Project can be a Pioneer for other similar Projects, according to the Minister. Reykjavik. Recuperado de: http://www.iceida.is/ english/about-iceida/news/nr/2251

Jiménez, P. (2016). Interpretando las perspectivas del desarrollo sustentable. En Hernández, Abraham, González, Héctor y Tamez, Gerardo (Eds.), Desarrollo Sustentable: de la teoría a la práctica (pp. 1336). Monterrey, México: Ediciones De Laurel.

Jimenez, S. (2011). Energía Renovable No Convencional: Políticas de Promoción en Chile y el Mundo. Libertad y Desarrollo, Serie Informe Económico. Núm. 218. Chile.

Revista Política, Globalidad y Ciudadanía, Vol. 3 No. 6, Julio - Diciembre 2017, Universidad Autónoma de Nuevo León, Monterrey, México Monterrey, México, ISSN 2395-8448. pp 62-72. http://revpoliticas.uanl.mx/index.php/RPGyC/article/view/80 
Parry, M.; Arnell, N.; Berry, P; Dodman, D.; Fankhauser, S.; Hope, C.; Kovats, S.; Nicholls, R., Satterthwaite, D., Tin, R.; Wheeler, T. (2009). Assessing the Costs of Adaptation to Climate Change: A Review of the UNFCCC and Other Recent Estimates, International Institute for Environment and Development and Grantham Institute for Climate Change, London.

Pristupa, A. y Mol, A. (2015). Renewable energy in Russia: The take of in solid bioenergy? Renewable and Sustainable Energy Reviews. Elsevier. Wageningen, The Netherlands. Recuperado de: https:// doi.org/10.1016/j.rser.2015.04.183

Republic of Estonia, Ministry of Economic Affairs and Communications (2015). Estonia-First in the Renewable Energy Cooperation Mechanisms. Recuperado de: https://admin.4energia.ee/wp-content/ uploads/2015/11/Estonia-first-mover-in-RES-Cooperation-Mechanisms-03.06.2015_all.pdf

OCDE (1999). Énergie: Les Prochaines Cinquante Années. Paris. Recuperado de: https://www.oecd.org/ $\mathrm{fr} / \mathrm{sti} /$ prospective/35635568.pdf

OECD (2017), OECD Development Co-operation Peer Reviews: Iceland 2017, OECD Publishing, Paris. Recuperado de: http://dx.doi.org/10.1787/9789264274334-en

Pasqualino, J.; Cabrera, C.; Vanegas Chamorro, M. (2015). Los impactos ambientales de la implementación de las energías eólica y solar en el Caribe Colombiana. PROSPECTIVA, 13 (1), 6875 .

World Energy Council (2014). Consejo Mundial de Energía. Recuperado de: https://www.worldenergy. org/wpcontent/uploads/2014/04/WEC_16_page_document_21.3.14_ES_FINAL.pdf 